\title{
Enhancing Team Performance Using Neurophysiologic Synchronies in a Virtual Training Environment
}

\author{
Marianne Clark ${ }^{1}$, Kimberly Cellucci ${ }^{1}$, Chris Berka ${ }^{2}$, Daniel J. Levendowski ${ }^{2}$, \\ Jonny Trejo ${ }^{2}$, Amy Kruse ${ }^{3}$, and Ron Stevens ${ }^{4}$ \\ ${ }^{1}$ Scientific Research Corporation, 1101 Remount Rd, Suite 500, Charleston, SC, 29406 \\ ${ }^{2}$ Advanced Brain Monitoring, Inc. 2237 Faraday Avenue, Suite 100, Carlsbad, CA, 92008 \\ ${ }^{3}$ Total Immersion Software 2001 Jefferson Davis Highway, Suite 703, Arlington, VA 22202 \\ ${ }^{4}$ IMMEX Project/UCLA Los Angeles, CA 90030 \\ mclark@scires.com, \{chris, dan, jtrejo\}@b-alert.com, \\ akruse@totimm.com, ron@immex.com
}

\begin{abstract}
A study was conducted to investigate the use of neurophysiologic synchronies as a measurement of team cognition (1) in a military-style virtual environment simulation. Neurophysiologic synchronies (NS), defined as the second-by-second quantitative co-expression of the levels of cognitive measures by individual members of a team (8), were found to be useful in monitoring the quality of teamwork and to be a means to identify more optimal patterns of team interaction which can be used to provide feedback during training. In the current study, findings showed promise for further research in the collection of NS. A framework is also proposed to support the research and training of team cognition.
\end{abstract}

Keywords: Team performance, team cognition, shared mental models, collaboration, neurophysiologic synchronies, electroencephalography (EEG), virtual environments, mission rehearsal training, RealWorld.

\section{Introduction}

Team performance is critical to the success of many organizations whether the teams are co-located or virtual; hierarchical or decentralized. Today, team performance is equally critical for our warfighter. Mission success is dependent upon the coordinated effort of those teammates executing the mission side-by-side as well as the efforts of the extended team responsible for planning and supporting the mission.

Our service men and women are well trained before they deploy. They are trained in how to operate their equipment, trained in operations and other requisite skills. However there is an identified gap between their formal training and the skills needed during a mission in a forward location. Sometimes what they need to know for a successful mission is only discovered the day prior.

This gap is being addressed by the Services via deployed mission rehearsal training. During these training exercises, specific, current tactics, threats and situations can be rehearsed and practiced prior to the mission. Critique of the training rehearsal can be 
given prior to the mission and a level of mission expertise can be attained by the team prior to the beginning of the mission.

Deployed mission rehearsal training provides an opportunity to address the complexity of team performance. One aspect of team performance is experience. Dyer (2) suggests that when a team has more experience working together, they are better able to coordinate their efforts, and have better team processes and performance. Klein, Zsambok and Thordsen (3) suggest that teams may have maturity levels as they move from entry-level to expert-level team. Mission rehearsal provides an opportunity to execute scenarios of potential situations to allow the teams to move from entry-level to expert.

A component of team expertise is addressed in the construct of team cognition or sometimes referred to as team level macrocognition $(4,5$, and 1). This construct can be seen as the integrated thinking of a team that includes cognition, behavior and attitudes that contribute to team performance. It includes shared knowledge or mental models and shared processes. Blickensderfer et al (6) distinguish between preperformance shared knowledge (mental model) and the dynamic shared knowledge, such as shared situation awareness, that forms among team members while performing a task. Klein (7) lists real-time team processes whose quality contributes to team performance, such as controlling the flow of information, forming shared situation awareness, applying strategies for decision making and problem solving and monitoring team performance.

Klein (7) and Blickensderfer et al. (6) suggest cognitive task analysis techniques to identify the knowledge requirements, including processes, decisions, barriers, errors, cues and strategies that need to be shared for effective team performance. Team cognitive task analysis techniques can be used to define the content of the shared mental model which will be the basis for mission training, including: mission objective, unique mission situation, roles and responsibilities, possible threats, and modifications to procedures for response to threats based upon latest intelligence and intent.

The use of a virtual training environment simulation of the mission provides the rehearsal needed to build the expertise of the current team and to create a team shared mental model when directed feedback can be provided. Mathieu et al. (5) used a networked simulation with six scenarios to investigate the development of shared mental models and their effect on team performance. They did not provide detailed analysis and feedback, such as an After Action Review, after each scenario because they were looking at the effects of experience alone. They found some improvement to coordination and cooperation, but it did not result in a greater shared mental model. Their findings support the idea that guided experience and developmental feedback is needed to support this type of learning. Given that team cognition addresses both internal and external processes of the team, a challenge to providing the most appropriate feedback to enhance training becomes identifying a measurement of team performance that allows for timely feedback to the team to guide them in their internal process of building their shared mental model and improving their team processes. Stevens et al. (8) suggests that internal processes can be studied via surrogate quantitative measures such as EEG metrics, pupil size, and heart rate variability. 


\section{Neurophysiologic Measurement of Team Performance}

Previous research examined the utility of collecting team members' electroencephalography (EEG) and electrocardiography (ECG) to measure cognitive components such as attention, workload, engagement, and stress for the modeling of team cognition (8). In this study, five three- person- teams of college students were asked to do a problem solving task to make a determination if the person shown in a simulated reality show should seek help for drug abuse. EEG data collected using the B-Alert wireless headset and software from Advanced Brain Monitoring, Inc (ABM). first decontaminates and then performs real-time calculations of cognitive states changes. The result is a value for mental work load (WL) and engagement (E) ranging from 0.1 to 1.0 for each 1 second epoch. The WL and $\mathrm{E}$ values are then normalized, values for each team are provided to a self organizing artificial neural network which then provides patterns of WL and $\mathrm{E}$ for each team, called neurophysiologic synchronies (NS). Neurophysiologic synchronies (NS) are the second-by-second quantitative co-expression of the levels of cognitive measures by individual members of a team. Stevens et al. (8) report unique neurophysiologic synchronies that were commonly found across the teams. Examples are patterns where all team members are actively engaged with moderate to high workload values to ones where one or more team members were not engaged and had low workload values.

Each team was tested against the identified patterns and it was determined that each team was different in the number of NS and which NS were most frequent. For example, Group 3 had fewer NS frequently repeated and those were ones where all teammates were engaged and working. At the other end of the spectrum was Group 2 with a high number of NS, with more of them representing team members who were not engaged or not working. Group 3 performed better based upon subjective ratings of video logs and objective measure of time to solution and correct solution. Group 2 took the longest to complete the task. Stevens et al. (8) further aligned the NS to tasks the operators were performing and how those tasks map to three processes: mental model formation, mental model sharing and integration, and mental model convergence and revision. They identified three NS whose patterns of reoccurrence aligned well to each process.

These findings suggest a promising approach for monitoring the quality of team internal processes that relate to team performance. We wanted to apply this approach to small teams of warfighters and to hopefully identify points to recommend more optimal synchronies after a mission rehearsal in order to improve team cognition and performance.

\section{Pilot Study}

A preliminary study was designed to further investigate the nature of team cognition, in particular, aspects of neurophysiologic synchronies as applied to small groups of warfighters. While the end objective is to look at team cognition at multiple levels: 1) the small team and 2) the multi-team, such as platoon level team, and to identify a method for providing directed feedback on internal team processes during a mission rehearsal session, the initial pilot study was designed to begin more simply. 
Our hypothesis is that as members of a team perform a collaborative task, each team member will generate varying degrees of cognitive components such as attention, workload and engagement and that the levels of these will vary depending upon the task and the level of expertise of the team. Specifically, we expect to see changes in the levels of the cognitive component from first execution of the scenario to the sixth execution. We expect to see improvements in team performance in terms of time to execute the scenario and ability to recognize and avoid threats from the first execution of the scenario to the sixth execution. We expect to see patterns in neurophysiologic synchronies change from the first execution of the scenario to the sixth execution.

\subsection{Tasks and Methods}

For this study, the training scenario was built to investigate the fundamentals of threeperson team collaboration in a dismounted engagement in a small village. Total Immersion Software, Inc. RealWorld software was used to construct the scenario. RealWorld is a PC-based simulation platform that enables non-programmers to rapidly build $3 \mathrm{D}$, geo-specific simulations. The primary team objective is to eliminate a foe and complete the mission, but to do that they must traverse a village and overcome unexpected obstacles. The scenario was designed to create cause-and-effect across team members. For example, if one team member did not obtain and communicate intelligence in a timely manner, the other team member would advance too far. This would trigger a hostile attack, a detonation of an IED, etc. Figure 1 shows a view of the village with potential threats identified. Team member A (route shown in red) and B (route shown in blue) were on the ground with a choice of routes to achieve their objective and meet at the rendezvous point(s). Team member $\mathrm{C}$ acted in a command and control role from a remote location while using a UAV (free camera) feed to gain his tactical oversight of the village and mission. His second role is to be the communication link for the team. He has full access to beyond-line-ofsight communications to provide intelligence reports of late breaking news to the team and to pass on requests for support from the team.

Six subjects were recruited to participate in the study. Testing was conducted at the ABM offices located in Carlsbad, Ca. Three gaming systems were set side-by-side in a test room. Subjects were assigned to role of team member A, B, or C.

The three team members were fitted with the ABM B-Alert EEG 9-channel wireless headset that has the following sensor site locations: F3, F4, C3, C4, P3, P4, $\mathrm{Fz}, \mathrm{Cz}$, and POz. An individual EEG baseline was collected. Subjects were given a 30- minute practice session to allow them to become familiar with the RealWorld gaming controls. The subjects were briefed on their mission and a provided map of the village. The scenario was then initiated. Duration of each scenario was approximately five minutes. Subjects participated in 6 sessions running the scenarios with a 10 minute break between each session. Subject's sessions were video recorded. Scenario start and end times and key event times (such as the triggering of an IED) were logged to RealWorld event log.

The EEG data was processed by the software from ABM which first decontaminates and then performs real-time calculations of cognitive state changes. 
Values for mental workload (WL) and engagement (E) for each individual team member was provided in a range from 0.1 to 1.0 for each 1 second epoch. Please refer to Berka et al. $(9,10$ and 11) for a detailed description of the development of the gauges and this process. WL and $\mathrm{E}$ values were then normalized for each individual team member, and then combined at each epoch for every team member into a vector representing the state of the team as a whole. The epoch-by-epoch team vectors were then presented to a self organizing artificial neural network developed by the IMMEX Project, which results in a series of neurophysiologic synchronies (NS).

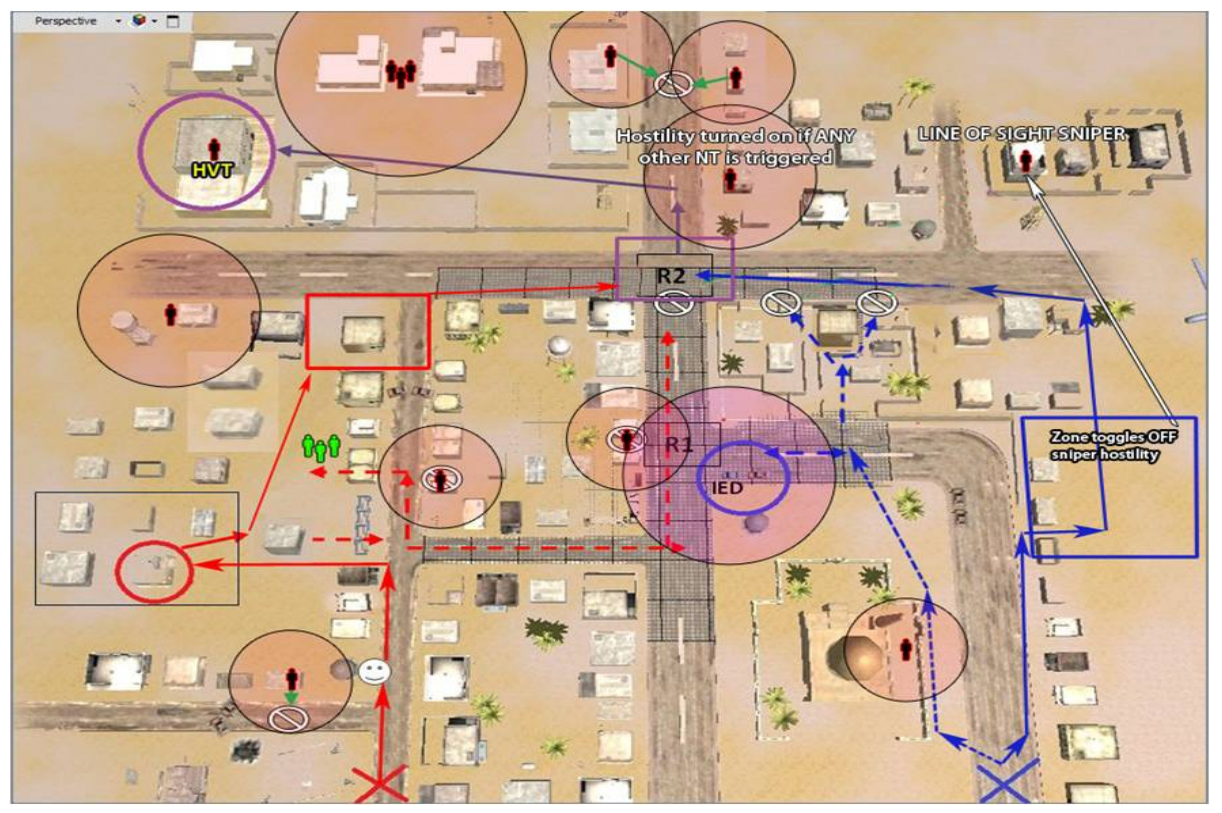

Fig. 1. Map of Village with Threats and Possible Routes

\subsection{Results and Discussion}

Preliminary analysis of the data was both encouraging and disappointing. Time to complete the scenario was reduced by 59\% from session 1 to session 2, but all subsequent sessions were no different from session 2 . This suggests that although the scenario had a dynamic quality, given that many of the threats could be triggered by proximity, the actual execution of the scenario lacked the complexity necessary to distinguish or allow for improvements of the team after the second trial.

The EEG data analysis was encouraging. Six neurophysiologic synchronies were frequently identified among the two teams. These can be characterized as the following:

- Team member A highly engaged, but B and $\mathrm{C}$ had low engagement

- Team members A and B were highly engaged 
- Team member $\mathrm{A}$ and $\mathrm{C}$ were highly engaged

- Team member B and C were highly engaged

- No team members were highly engaged

These NS occurrences are displayed over the course of a scenario in Figure 2. These invite the opportunity to investigate a relationship among operator tasks and team processes to NS as was done by Stevens et al. (8).

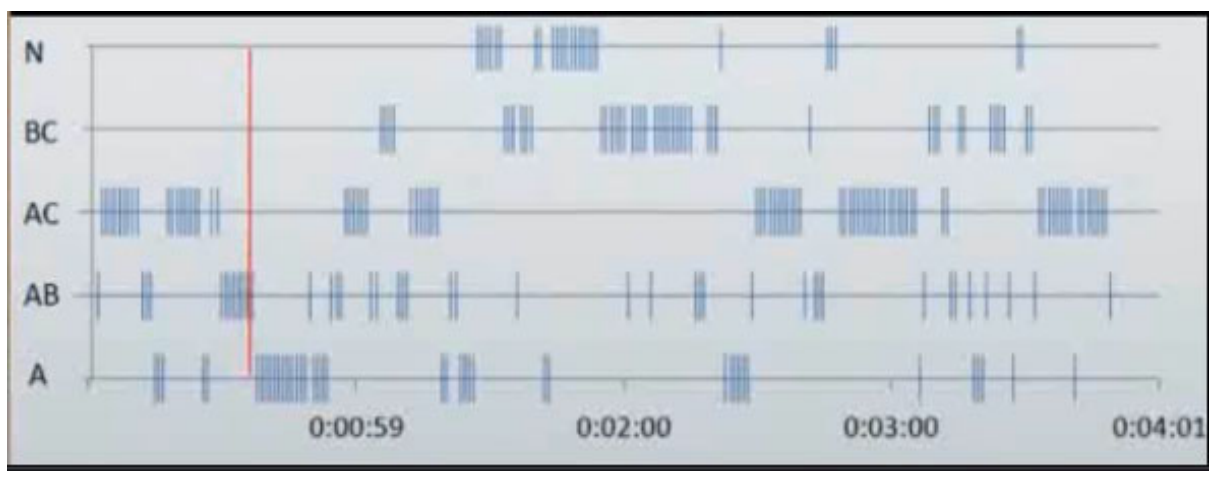

Fig. 2. Neurophysiologic Synchronies during a Scenario

Team engagement values were compared from session 1 to session 2 . All three team members had a $6 \%$ increase in the occurrence of low levels of engagement (this is the None NS described above) in session 2 when compared to session 1. Said another way, team members spend $6 \%$ more time not engaged in session 2 compared to session 1 . Also, the amount of time Team member $\mathrm{C}$ was highly engaged with either Team Member A or Team member B decreased by $6 \%$ from session 1 to session 2 .

While the difficulties encountered with the scenario resulted in limitations in the data collected, two conclusions can comfortably be reached. First, neurophysiologic synchronies can be identified in a military-type scenario using a virtual environment simulation. This suggests that with further refinement of methodologies and future research, it may be possible to measure NS, correlate them to tasks and internal processes, identify optimal and suboptimal NS demonstrated by performance, and provide this feedback in a form for training during deployed mission rehearsal.

A second conclusion that can comfortably be reached is that as new tools and techniques are discovered, a frequent challenge for cognitive researchers is to establish an experimental system that integrates these tools and techniques in a way that acknowledges the complexity of human cognition while allowing for experimental manipulation. Team cognition relies on a sophisticated understanding of shared mental models and the real-time monitoring and analysis of internal cognitive processes which must be tied to external performance. The number and types of human internal and external data points necessary to represent this complex phenomenon is in itself complex. In an attempt to address this fundamental research, a framework is proposed in the following section. 


\section{Proposed Experimental Framework}

In studying team cognition, as well as other complex phenomenon involving human cognitive abilities, the idea of an experimental platform has started to take form (12). Previous researchers in the field have developed systems to manage the research process, as well as the training process. This framework hopes to capitalize on these prior systems by use of modular plug-in components and defined interfaces. This will allow the "best of breed" tools and systems to be integrated into the framework.

The EXpertise transferred to novice Performance for Evaluation, Research and Training (EXPERT) framework is designed to be scalable and flexible which could provide a working platform for Cognitive Research, Training as well as Adaptive User Interfaces and Human Factors/User Interface evaluation.

As discussed in sections 1 and 2 above, both the delivery of a deployable mission rehearsal training system with access to targeted feedback for both internal and external process contributing to team performance, as well as the research platform needed to reach the point of such a deployable system requires the flow and integration of data that is easy for non-programmers to operate. The components shown in Figure 3 would make up such a system.

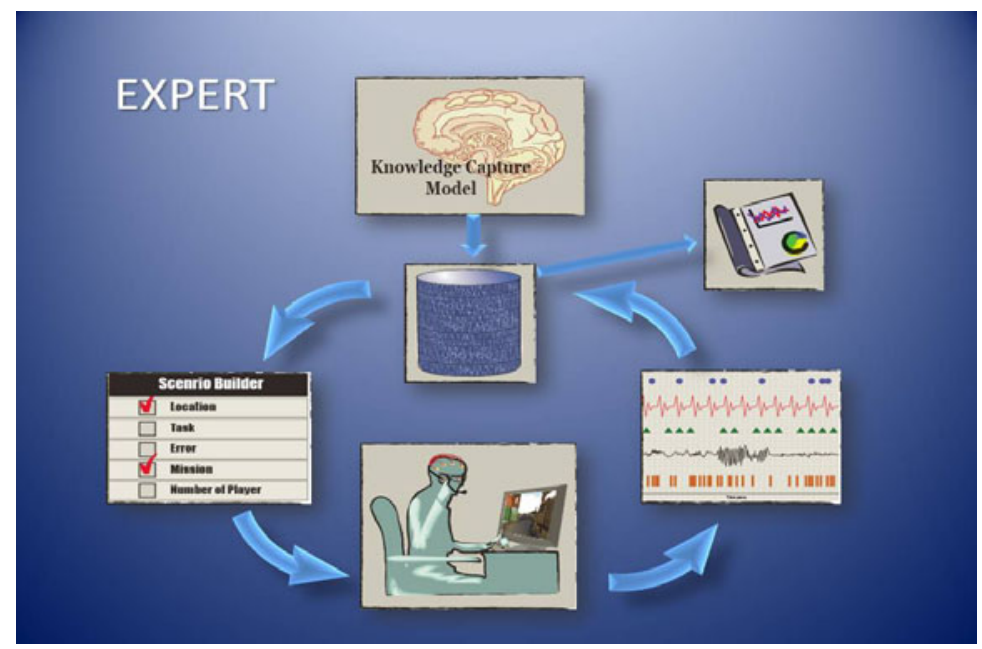

Fig. 3. EXPERT Framework Components

\subsection{Knowledge Capture and Cognitive Model}

Desired or expert knowledge, processes, and procedures need to be captured, edited regularly and understood by the expert and training staff. The ability to systematically capture and represent this expert model allows the model to become a foundational piece to the EXPERT framework to track variations from the model during scenario 
execution and provide a basis for establishing "success" criteria for a study. Variations may represent errors or at least events of interest to the study at hand whereas adherence to the model may represent confirmation or achievement of cognitive milestones.

\subsection{Database}

The database will provide the storage for the expert cognitive model as well as the operator/subject testing data that is stored in SCORM compliant format. This will allow the tracking of user progress toward objectives as well as the training record that could interface with a Learning Management System, if desired. The database will also provide the content required by the Mitigation component.

\subsection{Scenario Builder}

This component is the intersection of the cognitive model, the presentation system and the training objectives. It will contain a user interface that allows a trainer, tester or experimenter to specify the characteristics of the scenario to be run. Multiple scenarios can be created, stored and accessed by the testing component (for sequencing, etc). The builder will pass those values to the presentation system and also link to the knowledge model to draw the knowledge and behaviors appropriate for target participants and the OPFOR avatars. Scenario characteristics, such as the following need to be extracted and presented in a "checklist" format: pre-conditions, equipment load out, location and related map to be brought into presentation component, mission or tasking order details, personnel players, OPFOR personnel, and errors, threats, and situations of interest.

\subsection{Presentation System}

Presentations can include prototypes or already developed systems, training presentations such as simulations or serious games, or specially developed presentations for research studies. Outputs presented to the participant include sound, smell, facial features, virtual 3-D, and haptic sensations. Input devices to the system will include keyboard, mouse, joystick, touch, and gesture.

\subsection{Data Collection}

The framework will allow the collection of a variety of participant and system outputs for analysis to form the basis for understanding the current student/team model.

- Neurophysiologic sensors data from systems such as EEG, EKG, GSR

- Eye movement, fixations and pupilometry via eye trackers

- Participant facial responses

- Communication both verbal and chat

- Critical system events such as the presentation of cues as well as user's actions taken within the system 
- Performance measures. This may require an input module to prompt the participant for a response or may require correlation to the model to monitor performance.

- Synchronized logging of system events, sensor data, and user performance is stored and made available to the framework.

\subsection{Mitigation}

This component will compare the student model with the expert model. It will contain the rules for when a presentation intervention/change is required based upon participant and system data. It will also point to the content (remediation information, displays, etc) needed to address the mitigation.

As our research continues our goal is to build out this framework.

Acknowledgements. The development of the IMMEX ANN was supported by The Defense Advanced Research Projects Agency under contract number(s) NBCHC070101, NBCHC090054. The views, opinions, and/or findings contained in this article/presentation are those of the authors and should not be interpreted as representing the official views or policies, either expressed or implied, of the Defense Advanced Research Projects Agency or the Department of Defense.

\section{References}

1. Warner, N., Letsky, M., Cowen, M.: Cognitive Model of Team Collaboration: MacroCognitive Focus. In: Proceedings of the 49th HFES Annual Meeting, Orlando, FL, September 26-30 (2005)

2. Dyer, J.L.: Team research and team training: A state-of-the-art review. In: Muckler, F.A. (ed.) Human Factors Review, pp. 285-323. Human Factors Society, Santa Monica (1984)

3. Klein, G.A., Zsambok, C.E., Thordsen, M.L.: Team decision training: Five myths and a model. Military Review, 36-42 (1993)

4. Cooke, N., Kiekel, P., Salas, E., Stout, R.: Measuring team knowledge: A window to the cognitive underpinnings of team performance. Journal of Applied Psychology 7, 179-199 (2003)

5. Mathieu, J., Heffner, T., Goodwin, G., Salas, E., Cannon-Bowers, J.: The influence of shared mental models on team process and performance. Journal of Applied Psychology 85(2), 273283 (2000), doi:10.1037/0021-9010.85.2.273

6. Blickensderfer, E., Cannon-Bowers, J.A., Salas, E., Baker, D.P.: Analyzing Knowledge Requirements in Team Tasks. In: Schraagan, J., Chipman, S., Shalin, V. (eds.) Cognitive Task Analysis, Mahwah, NJ, pp. 431-447 (2000)

7. Klein, G.: Cognitive Task Analysis of Teams. In: Schraagan, J., Chipman, S., Shalin, V. (eds.) Cognitive Task Analysis, Mahwah, NJ, pp. 417-429 (2000)

8. Stevens, R.H., Galloway, T., Berka, C., Sprang, M.: Can Neurophysiologic Synchronies Be Detected during Collaborative Teamwork? In: Proceedings: HCI International 2009, San Diego, CA, July 19-24, pp. 271-275 (2009)

9. Berka, C., Levendowski, D., Cventinovic, M., Petrovic, M., Davis, G., Lumicao, M., Zivkovic, V., Popovic, M., Olmstead, R.: Real-time Analysis of EEG Indices of Alertness, Cognition, and Memory with a Wireless EEG Headset. International Journal of HumanComputer Interaction 17(2), 11-170 (2004) 
10. Berka, C., Levendowski, D.J., Ramsey, C.K., Davis, G., Lumicao, M.N., Stanney, K., Reeves, L., Regli, S., Tremoulet, P.D., Stibler, K.: Evaluation of an EEG-Workload Model in an Aegis Simulation. In Biomonitoring for Physiological and Cognitive Performance during Military Operations. In: Caldwell, J.A., Wesensten, N.J. (eds.) Proceedings of the International Society for Optical Engineering, vol. 5797, pp. 90-99 (2005)

11. Berka, C., Levendowski, D.J., Lumicao, M.N., Yau, A., Davis, G., Zivkovic, V.T., Olmstead, R.E., Tremoulet, P.D., Craven, P.L.: EEG Correlates of Task Engagement and Mental Workload in Vigilance, Learning and Memory Tasks. Aviation, Space, and Environmental Medicine 78(5) (2007)

12. Clark, M., Skinner, A., Kruse, A., Berka, C., Fidopiastis, C.: The Transfer of Learning from Virtual Reality Environments and Immersive Games to the Real World: Approaches and Challenges (2010) (unpublished paper) 